\title{
INVESTIGATION OF THE DISLOCATION SLIP ASSUMPTION ON FORMABILITY OF BCC SHEET METALS
}

\author{
Mariano. J. Serenelli, María A. Bertinetti and Javier W. Signorelli \\ Instituto de Física Rosario, (IFIR-CONICET), Universidad Nacional de Rosario \\ Bv. 27 de Febrero 210b, 2000 Rosario, Argentina, http://www ifir-conicet.gov.ar
}

Keywords: Formability, BCC material, Polycrystal yield surfaces.

\begin{abstract}
It is well known that the yield-surface shape greatly affects the prediction of strain-limit values, especially for biaxial-stretching paths. The sharpness of the material yield surface (PCYS) is related to the slip systems selected to accommodate the imposed deformation. Polycrystal models track the slip activity of each individual grain, allowing the evolution of anisotropy to be taken into account naturally. Consequently, we can correlate the change in the yield surface to the number and relative activity of the active slip systems.

In this study, we focus on how different plastic crystal-anisotropy assumptions influence the predicted formability strains. The forming-limit diagrams (FLDs) are calculated based on a ratedependent polycrystal model together with the Marciniak-Kuczynski (M-K) approach, which develops localized necking from an initial imperfection in the form of a narrow zone across the sheet.

Typically, numerical simulations of the FLDs performed for BCC sheets assume either 24 slip systems, of the type $\{110\}<111>$ and $\{112\}<111>$, or 48 slip systems, also including $\{123\}$ slip planes. Because the essential characteristics of multiple glide can be captured using 24 or 48 slip systems, researchers have been free to select either of the deformation modes for crystal plasticity simulations. However, when predicting FLD behavior with the full-constrain (FC) homogenization scheme, the selection of the active deformation modes strongly affects the calculated limit strains. For example, if two deformation modes are selected, the MK-FC model predicts unrealistically high limit strains in balanced-biaxial tension. These different behaviors can be avoided if a self-consistent (SC) homogenization scheme is used instead of the classical FC approach. The discrepancy between the MK-FC and MK-SC assumptions can be understood in terms of the differences in the slip systems selected by each case and, consequently, in the predicted lattice rotation and local curvature of the yield locus.
\end{abstract}




\section{INTRODUCTION}

Since the concept of the forming-limit diagram (FLD) was introduced by Keeler in 1961, this realistic and general method for analyzing plastic instability has been widely used for studying the formability of sheet metals. Through recent advances, the theoretical analysis of plastic instability has the potential to predict the near failure conditions measured by Keeler's technique. Most theoretical studies to predict FLD behavior are based on a model where the strain instability appears in the deformation process due to an imperfection already present in the material (Marciniak and Kuczynski, 1967). Numerous authors have adopted the Marciniak and Kuczynski model (M-K from now on) to describe strain localization. For the FLD simulations, crystallographic effects are taken into account by combining the $\mathrm{M}-\mathrm{K}$ approach and a viscoplastic crystal-plasticity model (MK-VPSC) (Signorelli, 2006; Signorelli et. al., 2009). Based on this approach, we have examined several factors that influence limit strains, such as hardening behavior and plastic anisotropy (Wu et al., 2004; Inal et al., 2005; Signorelli et. al., 2009). It is widely recognized that the crystallographic texture strongly affects forming-limit diagrams and the macroscopic anisotropy of polycrystalline sheet metals. Recent work has emphasized characterizing and quantifying the effect of an initial cube texture and its evolution on the predictions of forming limit diagrams (Signorelli and Bertinetti, 2009).

It has long been recognized that large-strain deformations lead to changes in the radius and shape of the stress potentials away from any given initial state. With a crystal-plasticity based description of mechanical deformation that accounts for the development of preferred crystallographic orientations via the rotation of individual grains and the natural evolution of slip resistance, we can follow the evolution of the yield surface in polycrystal materials. If the multiple glide available in BCC materials, two or three deformation modes, is included in the calculation, we can also investigate how this ambiguity affects FLD predictions. In what follows, two types of slip modes are considered: (i) a BCC crystal with 24 slip systems of the types $\{110\}<111>$ and $\{112\}<111>$ (referred to as BCC24); and (ii) a BCC crystal with 48 slip systems of the types $\{110\}<111>,\{112\}<111>$ and $\{123\}<111>$ (called BCC48). In the BCC metal literature, many different choices of slip systems have been used. Often, the $\{123\}<111>$ slip systems have not been accounted for (Inal et al., 2005; Van Houtte et al., 2006; Li et al., 2007; Radhakrishnan and Sarma, 2008; Delannay et al., 2009; Franz et al., 2009). On the other hand, many authors employed BCC48 in their simulations (Raabe, 1995; Tóth et al., 1997; Choi, 2002; Nemat-Nasser et al., 1998).

The purpose of this work is to determine how the different slip plastic assumptions, BCC24 and BCC48, affect the yield-surface shape within the context of crystal viscoplasticity theory. Specifically, we are concerned with comparing the predictions of stress potentials obtained from the FC and SC models and seeing how these potentials influence the predicted limit strains.

\section{THEORETICAL FRAMEWORK}

\subsection{VPSC formulation}

This work adopts several assumptions for modeling material behavior. According to earlier contributions, a viscoplastic (VP) behavior is considered for the single crystal, and a selfconsistent (SC) homogenization scheme is used for the transition to a polycrystal (VPSC) response. We assume that plastic deformation is a result of crystallographic slip due to dislocation motion on the active slip systems. The dislocation slip rates, $\dot{\gamma}^{s}$, are derived from the resolved shear stresses, $\tau^{s}$, using a viscoplastic exponential law: 


$$
\dot{\gamma}^{s}=\dot{\gamma}_{o}^{s}\left|\frac{\tau^{s}}{\tau_{c}^{s}}\right|^{1 / m} \operatorname{sgn}\left(\tau^{s}\right)
$$

The label $s$ refers to the set of potential slip systems available in the material. Here, $m$ is the slip-rate sensitivity index, assumed to be constant during deformation and equal for all slip systems, $\dot{\gamma}_{o}$ is the reference shear rate and $\tau_{c}^{s}$ is the critical resolved shear stress, applied to each system. An isotropic hardening law is adopted, and the evolution of the critical shear stress is given by:

$$
\dot{\tau}_{\mathrm{c}}=\sum_{s} h^{s}\left|\dot{\gamma}^{s}\right|
$$

where, $h^{s}$ are the hardening moduli behaviors, which depend on $\gamma$ (accumulated sum of the single-slip contributions to $\gamma^{s}$ ). These moduli can be written using the initial hardening rate $h_{0}$ and the hardening exponent $n$ :

$$
h^{s}=h_{0}\left(\frac{h_{0} \gamma}{\tau_{\mathrm{c}}^{s} n}+1\right)^{n-1} ; \quad \gamma=\sum_{s} \int_{0}^{t}\left|\dot{\gamma}^{s}\right| d t
$$

The strain rate $\mathbf{d}$ and the shear rates $\dot{\gamma}^{s}$ are related as follows:

$$
\mathbf{d}=\sum_{s} \mathbf{m}^{s} \dot{\gamma}^{s}
$$

where, $\mathbf{m}^{s}$ is the Schmid tensor, which describes the geometry of the $s$ slip system in the single crystal. The macroscopic deviatoric stress $\overline{\mathbf{s}}$ and deviatoric strain rate $\overline{\mathbf{d}}$ of the material are obtained by averaging the local values $\mathbf{S}$ and $\mathbf{d}$, respectively, weighted by the volume fraction of crystal orientations. The relation between $\mathbf{d}$ and $\overline{\mathbf{d}}$ ( $\mathbf{s}$ and $\overline{\mathbf{s}}$ ) depends on the model assumptions. In our case, this model is based on the assumption that each grain is surrounded by a homogeneous effective medium that has the average properties of the polycrystal. Such a formulation leads to an interaction equation that linearly relates the stress and strain rate in the grain with the overall stress and strain rate of the effective medium. Basic discussions of this approach can be found in the literature (Molinari et al., 1987; Lebensohn and Tomé, 1993).

\subsection{The stress-potential function}

In the case of viscoplastic models, there is no yield surface in the classical sense but a yield potential can be defined, with a very similar role to the yield surface in rate-independent models. The shape of the yield potential gives direct information about the plastic anisotropy of the crystal and its size depends on the selected plastic work rate. The stress potential function $f(\overline{\mathbf{s}})$ is defined by a constant plastic work rate $\dot{W}_{0}$ along the potential. The plastic work corresponding to an arbitrary strain rate $\overline{\mathbf{d}}_{0}$ is defined as:

$$
f(\overline{\mathbf{s}})=\text { const. }=\overline{\mathbf{s}}: \overline{\mathbf{d}}=\overline{\mathbf{s}}_{0}: \overline{\mathbf{d}}_{0}=\dot{W}_{0}
$$

where, $\overline{\mathbf{s}}_{0}$ is the stress state corresponding to $\overline{\mathbf{d}}_{0}$. In the viscoplastic scheme used in this work $\overline{\mathbf{d}}$ is prescribed and $\overline{\mathbf{s}}$ is determined. As $\overline{\mathbf{s}}$ is not known a priori, the obtained plastic potential rate can be different from $\dot{W}_{0}$, i.e. the stress point does not necessarily lie on the 
selected equipotential. When both the imposed strain rate and the obtained stress state are deviatoric, the plastic work rate lies on another equipotential contour. The desired stress and strain rate on the selected equipotential can be obtained by following Hutchinson's method (Hutchinson, 1976). He showed that when the magnitude of the strain rate is changed by a factor $\lambda$, the stress response of the polycrystal gives:

$$
\overline{\mathbf{s}}(\lambda \overline{\mathbf{d}})=\lambda^{m} \overline{\mathbf{s}}(\overline{\mathbf{d}}) .
$$

As a consequence, the magnitude can be scaled as follows:

$$
\overline{\mathbf{s}}^{*}=\left(\frac{\dot{W}_{0}}{\dot{W}}\right)^{m / 1+m} \overline{\mathbf{s}} ; \quad \overline{\mathbf{d}}^{*}=\left(\frac{\dot{W}_{0}}{\dot{W}}\right)^{1 / 1+m} \overline{\mathbf{d}} .
$$

The description of the complete yield locus requires probing all possible test directions. However, in this study, we will focus on the $\sigma_{11}-\sigma_{22}$ projection of this locus, due to the assumption of plane stress, $\sigma_{33}=0$. For computing the $\sigma_{11}-\sigma_{22}$ Cauchy states, different strain-rate boundary conditions, in the global reference frame, are imposed appropriately to obtain different points on the yield potentials. Then, all possible directions in the strain-rate sub-space are scanned in steps of one degree.

\section{RESULTS}

In persuing our analysis of how the $\mathrm{BCC} 24$ and $\mathrm{BCC} 48$ deformation modes affect yield surfaces and FLDS, we assume the same initial random texture, as described by 1000 equiaxed grains. All numerical simulations are performed using both, FC and SC, homogenization schemes. Following Inal's procedure (Inal et al., 2005), we take the same uniaxial stress-strain curve fit for all cases. Accordingly, the hardening parameters are chosen to give identical uniaxial-stress responses. We take the BCC48-FC's stress-strain curve as a reference (see Table 1). For the calculations, the slip resistances, $\tau_{\mathrm{c}}^{s}$, of all slip systems are taken equal to one another; the rate sensitivity is $m=0.02$, and the reference slip rate is $\dot{\gamma}_{o}^{s}=$ $0.001 \mathrm{~s}^{-1}$.

\begin{tabular}{|l|c|c|c|c|}
\hline Material & \multicolumn{2}{|c|}{ BCC48 } & \multicolumn{2}{c|}{ BCC24 } \\
\hline Scheme & FC & VPSC & FC & VPSC \\
\hline $\mathbf{h}_{\mathbf{0}}(\mathrm{GPa})$ & 0.808 & 0.808 & 0.795 & 0.980 \\
\hline $\mathbf{n}$ & 0.23 & 0.26 & 0.23 & 0.26 \\
\hline $\boldsymbol{\tau}_{\boldsymbol{c}}(\mathrm{GPa})$ & 0.0305 & 0.0400 & 0.0300 & 0.0370 \\
\hline
\end{tabular}

Table 1: Material parameters used in the simulations

The influence of the selection of the active deformation modes on the shape of yield potentials will be addressed first.

\subsection{Calculated polycrystal viscoplastic potentials}

As mentioned above, the yield potentials of the materials are calculated by imposing different plastic strain-rate tensors under a state of plane stress. The stress potentials are calculated in the $\sigma_{11}-\sigma_{22}$ section. The selected reference strain mode, before the yield-locus simulation, is that of equi-biaxial stretching up to a plastic strain $\varepsilon_{11}=0.32$. Figure 1a shows 
the theoretical yield loci predicted for $\mathrm{BCC} 24$ and $\mathrm{BCC} 48$ materials. The equi work-rate surfaces are normalized to the work-rate for uniaxial stretching, as calculated for BCC48-FC. Since the direction of the plastic-strain rate plays an important role in simulating the deformation process, the directions at different points along the predicted yield surfaces are analyzed. To characterize these vector directions two angles, $\theta$ and $\varphi$, as shown in Figure $1 \mathrm{~b}$, are defined in the Cauchy stress reference frame. Because all simulations begin with the same initially random texture, the computed yield potentials before stretching are the same. During plastic deformation, as a consequence of the texture development, the crystal orientation distributions change, and the yield loci also change. The $\{110\}$ pole figures after the equibiaxial loading are shown on the bottom in Figure 1a (BCC48-FC, BCC24-FC, BCC48-SC and BCC24-SC from left to right). The stress potentials predicted for the BCC24 and BCC48 materials exhibit a large difference between the FC and VPSC assumptions. In both materials, VPSC predicts larger yield stresses and sharper curvatures in the vicinity of equi-biaxial tension than the FC model. No differences can be seen when the calculations are carried out with the VPSC scheme, regardless of the material. But, for the FC calculations, although the predictions have similar shapes and tendencies, small differences occur in the vicinity of equibiaxial tension, where the local curvature of the BCC24 potential slightly decreases. These small changes in the shape of the yield locus, which can influence the stress response corresponding to an imposed strain-rate mode, can be seen in the detail of Figure 1b. These subtle variations are clearly depicted in the vicinity of $\varphi=45^{\circ}$. Upon close inspection, slopes generated by the BCC24 and BCC48 calculations differ in detail (open and close symbols respectively in the detail of Figure 1 b) reflecting a decrease in the sharpness of the yield locus. For the VPSC simulations, the strain-rate directions increase dramatically when compared with the FC calculations. Again, this behavior is directly associated with the sharper curvature of the VPSC stress potentials. In essence, Figure 1 shows the effects of texture evolution on the yield loci, as calculated by FC and VPSC homogenization schemes.
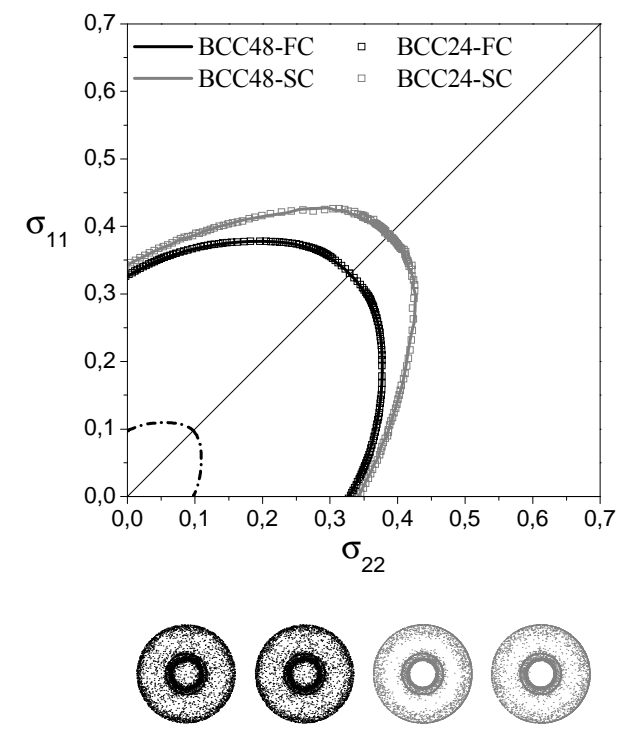

a)

Figure 1: a) Stress potentials of BCC24 and BCC48 deformation as computed with FC and VPSC homogenization schemes; b) Directions of the plastic strain rate vectors $\overline{\mathbf{d}}$.

According to Lian et al. (1989), the yield surface shape has a tremendous effect on the FLDs, and Neale and Chater (1998) demonstrated that a decrease in the sharpness of the 
stress potentials in equi-biaxial stretching is a factor promoting larger limit strains. A sharp curvature allows the material to quickly approach plane strain, and therefore, results in the prediction of a relatively low limit strain. Next, we analyze the effects of active slip-system selection on strain localization in biaxial stretching.

\subsection{Predicted forming limits}

We applied the MK-VPSC and MK-FC approaches for predicting limit strains in the present materials. For details of the implementation of the MK approach we refer the reader to Signorelli et al., 2009. The material is assumed to have an imperfection in the form of a groove or band that is initially inclined at an angle $\psi$ with respect to the transverse direction. The initial-thickness ratio inside and outside the band defines a parameter $f$, which must first be fixed. Its value is taken to be 0.99 for all FLD calculations in this work. To analyze the development of deformation localization during proportional straining, the calculations are performed over different strain paths in the biaxial zone. These strain paths are defined in terms of the strain ratio $\rho=\overline{\mathrm{d}}_{22} / \overline{\mathrm{d}}_{11}$ over the range $0 \leq \rho \leq 1$.

The predicted limit strains are presented in Figure 2. Results clearly illustrate large differences between the FC and VPSC homogenization schemes, particularly near equibiaxial stretching, regardless of the material. For each homogenization scheme, both materials reach the same values in plane strain. Within MK-VPSC, the profiles of the BCC48 and BCC24 simulations are similar over the range of strain ratios $0.2 \leq \rho \leq 0.6$, and slight differences can be seen near equi-biaxial stretching. On the other hand, within MK-FC, the strain values are similar as $\rho$ increases from 0.2 to 0.8 , but, for $\rho \geq 0.9$, critical values calculated with the BCC24 deformation model increase to a nonrealistic high value at $\rho=1$. The same response was found by Inal et al. (2005). In their work, they compared numerical simulations of FLDs based on a rate-dependent Taylor-type polycrystal-plasticity model for FCC and BCC sheets using 12 and 24 systems, respectively. For BCC24-FC deformation, they also predicted extremely high limit strains without texture evolution.

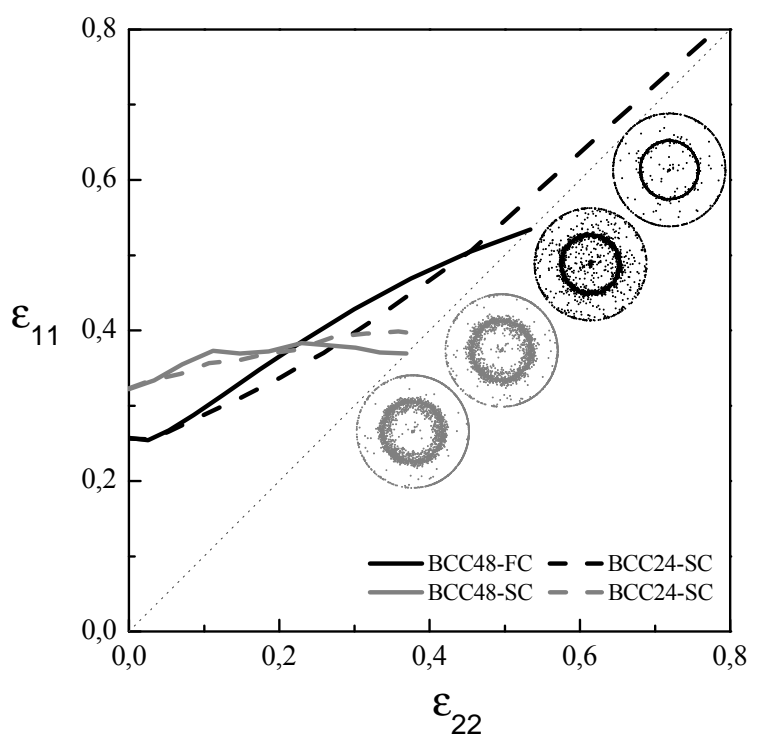

Figure 2: Influence of the active-selected modes on the FLD.

Figure 2 includes a plot of the textures of each material at the end of the equi-biaxial loading path. Clearly, BCC 24 and BCC48 textures evolve to different states, producing the 
strong effects observed in the FLD behavior.

In order to assess the effect of the yield-surface shape on the forming-limit behavior close to the balanced-biaxial stretching zone, we calculated the yield loci corresponding to each of the necking limit strains (Figure 3). The shapes and curvatures produced by the FC and VPSC models are quite different. Within the VPSC framework, the yield loci are sharper, and only small differences can be found between BCC 24 and BCC48 based simulations. This is consistent with the similar limit-strain values predicted by the VPSC model, as shown in Figure 2.

The differences are more pronounced for the $\mathrm{FC}$ calculations. The curvature of $\mathrm{BCC} 24$ yield locus is blunter than that of the BCC48, again in agreement with the predicted limit strains. As mentioned in the previous subsection, when calculations are carried out up to a plastic strain of 0.32 , before necking, only subtle variations are observed, as pointed out by Kuroda and Tvergaard (1999). Nevertheless, in their work, the authors claim that a quite reasonable representation can be obtained for BCC48 and BCC24 materials in the FC elastoplasticity framework (i.e. only yield-surface analysis was performed). Here, we found that this assessment does not strictly apply in the case of critical-strain calculations, especially close to equi-biaxial stretching paths.

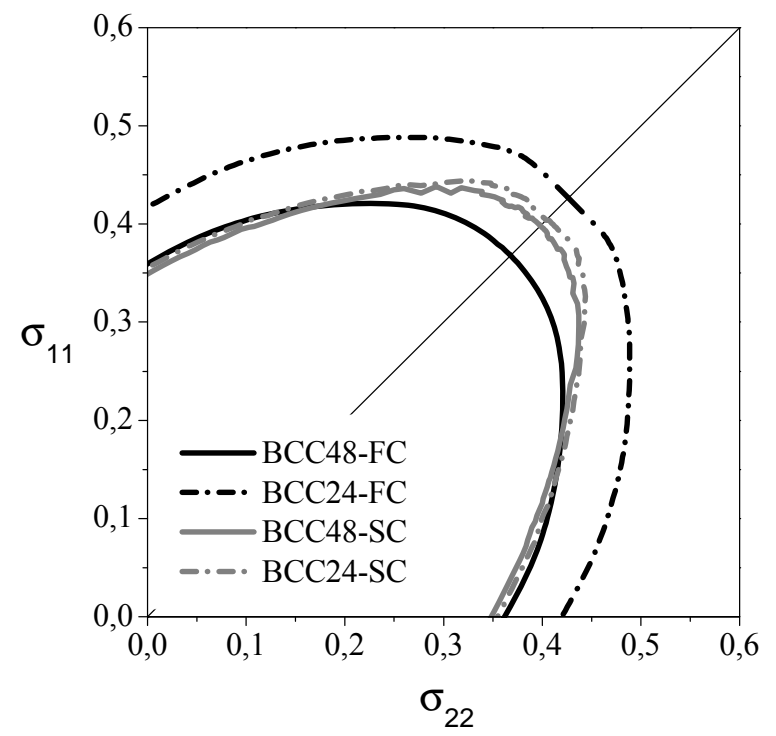

Figure 3: Stress potential at equi-biaxial failure for all tested materials.

Two other qualitatively similar ways of analyzing the stress potential are depicted in Figure 4. The directions of the plastic-strain rate at different points along the predicted yield surfaces and a yield-surface shape hardening diagram (Yoshida et al., 2007) are shown in Figure 4a-b, respectively. The latter represents a relationship between $\overline{\mathrm{d}}_{22} / \overline{\mathrm{d}}_{11}$ and $\sigma_{11} / \sigma_{11}^{\text {biax }}$ for different strain paths, where $\sigma_{11}^{\text {biax }}$ is defined as the $\sigma_{11}$ stress corresponding to $\overline{\mathrm{d}}_{22} / \overline{\mathrm{d}}_{11}=1$.

Differences in the sharpness of the stress potentials in equi-biaxial stretching are clearly illustrated in these figures. For the BCC24-SC and BCC48-SC deformation modes, the slight changes observed in the predicted critical-strain values are correlated to the similar profiles shown in Figure 4a-b. In the vicinity of $\varphi=45^{\circ}$, the sharpness of both yield loci are characterized by an abrupt change of $\theta$. Over the range $42^{\circ} \leq \varphi \leq 47^{\circ}, \theta$ varies linearly from $23^{\circ}$ to $63^{\circ}$, and that allows the material to quickly approach a plane-strain state with small 
variations of stress state. We attribute the reasonable description of the BCC material behavior using either 24 or 48 slip systems, to the capability of the VPSC model to assign different strain values to each grain. On the other hand, the curves calculated with the FC theory, in accordance with the observed yield loci, are not as steep and clearly different for the two materials. For the BCC24-FC approach the direction of the plastic strain-rate $\overline{\mathbf{d}}$, or $\theta$, seems nearly invariant in the vicinity of $\varphi=45^{\circ}$, which is consistent with the similarity of the yield surface curvatures. But, for BCC48-FC, the values of $\theta$ increase steadily with $\varphi$ over this range. These effects are shown in Figure $4 \mathrm{~b}$ Fig. $4 \mathrm{~b}$, together with the $p$-parameter (Barlat et al., 1989). This parameter is calculated as the ratio between the yield stress in planestrain, $\sigma_{11}$, to that in equi-biaxial tension, $\sigma_{11}^{\text {biax }}$. It should be noted that the trends in $p$ and the predicted limit-strain values are consistent with our analysis of the variation between FC and VPSC results. For VPSC simulations, the $p$-parameter has the same values. However, this is not the case when we compare BCC24-FC and BCC48-FC materials (i.e. note that the dashed and dotted line is below the solid line).

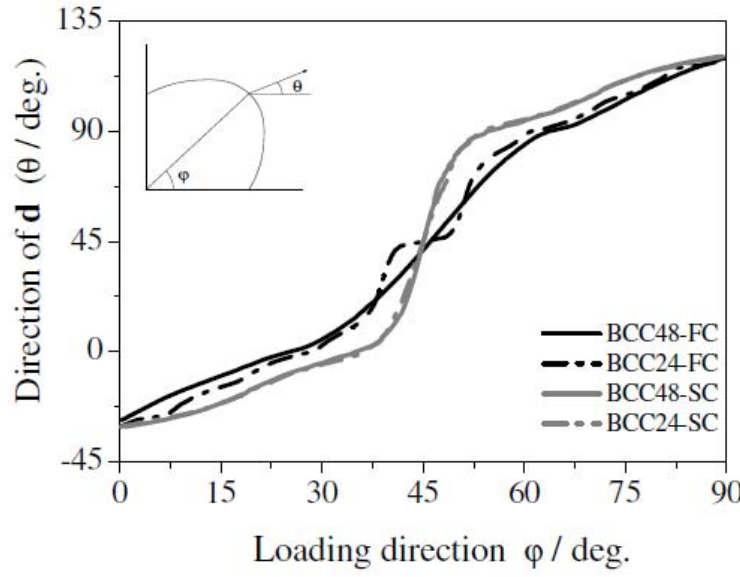

a)

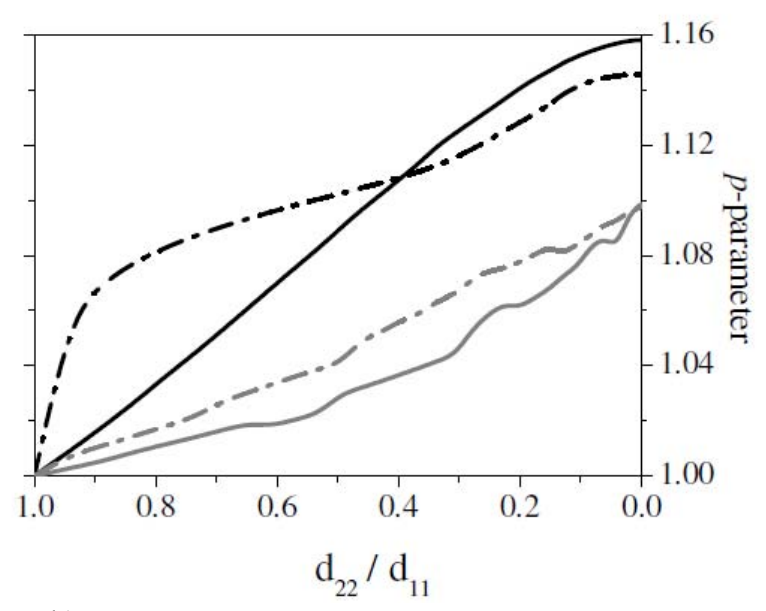

b)

Figure 4: a) Directions of the plastic strain rate vectors $\overline{\mathbf{d}}$; b) Diagram representing the relationship between $\overline{\mathrm{d}}_{22} / \overline{\mathrm{d}}_{11}$ and $\sigma_{11} / \sigma_{11}^{\text {biax }}$ for different paths (p-parameter).

\section{DISCUSION AND CONCLUSIONS}

As mentioned above, the differences in the relative active slip systems are one of the most relevant features for comparing FC and VPSC simulations. As a part of this study, we analyzed the predicted plastic activity in the equi-biaxial $\left(44^{\circ} \leq \theta \leq 46^{\circ}\right)$ strain-rate states that characterize the curvature of the yield locus. We found that in all the cases the critical strains are achieved when slip on the $\{110\}<111>$ systems decreases to a minimum. We found that for the BCC24-SC, BCC48-SC and BCC48-FC deformation modes the plastic activity and the average number and type of slip systems remain constant (i.e. independently of the theta value). However, interestingly, for the BCC24-FC slip model, the way in which deformation is accommodated depends on the theta value. The minimum value of activity on the $\{110\}<111>$ systems is found in the equi-biaxial state $\left(\varphi=45^{\circ}\right)$. While, moving away from the equi-biaxial state results in an increase in slip on these systems. We believe that, although the strain-rate path changes, due to the fact that different slip systems accommodate the deformation, the ratio $\sigma_{11} / \sigma_{22}$ remains constant, retarding the initialization of strain localization. 
With increasing deformations, the relative activity of the $\{110\}<111>$ slip systems diminishes while the $\{112\}<111>$ slip activity increases. If we include $\{123\}<111>$ slip as a potentially deformation mode, we find that its relative activity remains constant (at approximately 50 percent) to the flow instability, while, as previous observed, the $\{110\}<111>$ slip mode activity decreases to near zero at failure. We attribute this behavior to the fact that all slip resistances are equal to one another, and that for the same critically resolved shear strength, $\{123\}<111>$ slip tends to more easily accommodate the deformation (Tóth. et al., 1997, Signorelli et al. 2006).

In order to understand how the texture evolution affects the critical strains near the equibiaxial zone ( $\rho \geq 0.7)$ and shapes the yield locus, we repeated the FC calculations freezing the texture in a particular state. Figure 5 shows results for BCC24 and BCC48 deformation assuming an initial texture corresponding to the predicted texture at failure from the previous simulations (e.g., we ran BCC24-FC with the final texture predicted by the BCC24-FC or BCC48-FC simulations). Over the range $0.7 \leq \rho \leq 1.0$ the critical strain values are practically identical for simulations assuming 48 active slip systems, independent of the initial texture. However, for BCC24-FC, the tendency is quite different, and the limit-strain values reflect the influence of texture and a relationship to the polycrystal yield surface. This last effect is pronounced as we approach the equi-biaxial state, because the sharpness of the yield locus controls the limit-strain behavior.

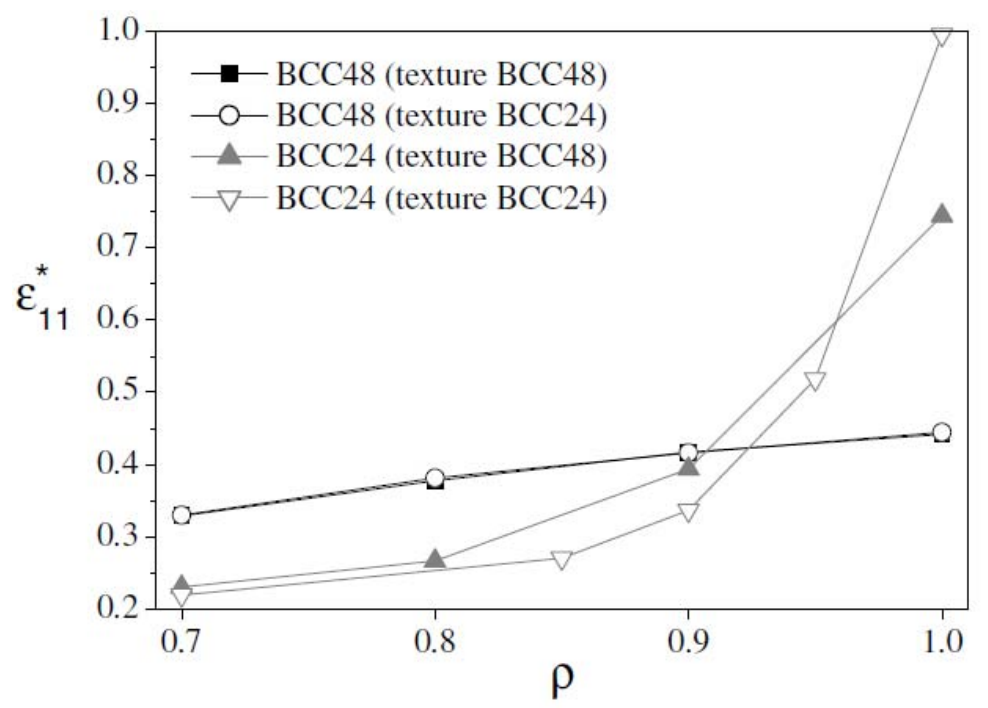

Figure 5: Influence of the plastic material representation on the MK-FC predictions close to equi-biaxial stretching.

In summary, although it is normally accepted that a BCC material can be represented using 24 or 48 slip systems, we demonstrated that the MK-FC FLD calculations are sensitive to the material plasticity assumption in the vicinity of equi-biaxial stretching. This, however, was not the case when we used a self-consistent transition scale for the homogenization procedure. For these calculations, we found that either 24 or 48 slip systems predicted similar FLD curves over the whole range of deformations. In order to understand this apparent ambiguity, we are now incorporating models of dislocation-based hardening in the constitutive law. This should provide a better description of the effects of microscopic-scale substructure on yield loci (Fourty et al., 2009). 


\section{REFERENCES}

Barlat, F. Forming limit diagrams-predictions based on some microstructural aspects of materials. In: Forming Limit Diagrams: Concepts, Methods and Applications, R.H. Wagoner, K.S. Chan and S.P. Keeler (Eds.), 275-302,1989.

Choi, S.H. Macroscopic texture and anisotropy in Ti-added IF steels, Materials Science Forum 408-412:1073-1078, 2002.

Delannay, L., Melchior, M.A., Signorelli, J.W., Remacle, J.-F., Kuwabara T. Influence of grain shape on the planar anisotropy of rolled steel sheets-evaluation of three models, Computational Materials Science, 45:739-743, 2009.

Franz, G., Abed-Meraim, F., Ben Zineb, T., Lemoine, X. and Berveiller, M. Strain localization analysis using a multiscale model, Computational Materials Science, 45:768773, 2009.

Fourty, A., Roatta, A., Bolmaro, R., Signorelli, J.W. Estudio de la subestructura de granos individuales en chapas de acero laminadas mediante un modelo de endurecimiento basado en densidad de dislocaciones, This conference, 2009.

Hutchinson, J.W. Bounds and self-consistent estimates for creep of polycrystalline materials, Proceeding R. Society London A, 348:101-108, 1976.

Inal, K., Neale, K.W. and Aboutajeddine, A. Forming Limit comparison for FCC and BCC sheets, International Journal of Plasticity, 21:1255-1266, 2005.

Keeler, S.P. Plastic instability and fracture in sheets stretched over rigid punches, Ph.D Thesis, Massachusetts Institute of Technology, Cambridge, MA, 1961.

Kuroda, M. and Tvergaard V. Use of abrupt strain path change for determining subsequent yield surface: illustrations of basic idea, Acta Materialia 47:3879-3890, 1999.

Lebensohn, R.A. and Tomé, C.N. A Self-Consistent Approach for the Simulation of Plastic Deformation and Texture Development of Polycrystals: Application to $\mathrm{Zr}$ alloys, Acta Metall. Mater. 41: 2611-2624, 1993.

Li, S., Gazder, A., Beyerlein, I. and Davies, C. and Pereloma, E. Microstructure and texture evolution during equal channel angular extrusion of interstitial-free steel: Effects of die angle and processing route, Acta Materialia, 55:1017-1032, 2007.

Lian, J., Barlat, F. and Baudelet, B. Plastic behavior and stretchability of sheet metals. II. Effect of yield surface shape on sheet forming limit, International Journal of Plasticity, 5:131-147, 1989.

Marciniak, Z. and Kuczynski, K. Limit strains in processes of stretch-forming sheet metal. International Journal of Mechanical Science, 9:609-620, 1967.

Molinari, A., Canova, G.T. and Ahzi, S. A Self-Consistent Approach of the Large Deformation Polycrystal Viscoplasticity, Acta Metall. 35, 2983-2994, 1987.

Neale, K.W. and Chater, E. Limit strain predictions for strain-rate sensitive anisotropic sheets, International Journal of Mechanical Science, 22:563-574, 1980.

Nemat-Nasser, S., Okinaka, . and Luqun, N. J. of Mechanical Physics Solids, 46 :1009-1016, 1998.

Raabe, D. Simulation of rolling textures of b.c.c, metals considering grain interactions and crystallographic slip on $\{110\},\{112\}$ and $\{123\}$ planes, Materials Science and Engineering A, 197:31-37, 1995.

Radhakrishnan, B. and Sarma G.B. Coupled simulations of texture evolution during deformation and recrystallization of fcc and bcc metals, Materials Science and Engineering A 494:73-79, 2008.

Signorelli, J.W. Predicción de la curva límite de formabilidad utilizando un modelo autoconsistente viscoplástico, Mecánica Computacional Vol XXV, 2082-2096, 2006. 
Signorelli, J.W., Bolmaro R., Turner, P., Bertinetti, M., Insausti, J., Lucaioli, A., García, C. and Iurman, L. Predicción de la curva límite de formabilidad en aceros utilizando plasticidad cristalina, Proceedings Congreso SAM-CONAMET 2006, CD (trabajo d3).

Signorelli, J.W., Bertinetti M.A., Turner P.A. Predictions of forming limit diagrams using a rate-dependent polycrystal self-consistent plasticity model, International Journal of Plasticity, 25:1-25, 2009.

Signorelli, J.W. and Bertinetti, M.A. On the role of constitutive model in the forming limit of FCC sheet metal with cube orientations, International Journal of Mechanical Sciences, 51: 473-480, 2009.

Tóth, L.S., Molinari, A. and Raabe, D. Modeling of rolling texture development in a ferritic chromium steel, Metallurgical and Materials Transactions A, 28:2343-2351, 1997.

Van Houtte, P., Kanjarla, A., Van Bael, A., Seefeldt, M. and Delannay L. Multiscale modelling of the plastic anisotropy and deformation texture of polycrystalline materials, European Journal of Mechanics A Solids, 25: 634-648, 2006.

Wu, P.D., MacEwen, S.R., Lloyd, D.J and Neale, K.W. Effect of Cube Texture on Sheet Metal Formability, Materials Science and Engineering A, 364:182-187, 2004.

Yoshida, K., Ishizaka, T., Kuroda M. and Ikawa, S. The Effects of Texture on Formability of Aluminum Alloy Sheets, Acta Materialia 55:4499-4506, 2007. 